\title{
Are We Able to Suppress Disease Activity Adequately in Patients With Established Rheumatoid Arthritis? An Observational and Cross-Sectional Study
}

\author{
İlker ŞENGÜL, Seniz AKÇAY YALBUZDAĞ, Buğra İNCE, Altınay Göksel KARATEPE, Taciser KAYA \\ Department of Physical Medicine and Rehabilitation, İzmir Bozyaka Training and Research Hospital, İzmir, Turkey
}

\begin{abstract}
Objectives: This study aims to explore current disease activity status and simultaneous pharmacological therapies in patients with established rheumatoid arthritis (RA) to determine the extent to which treatment targets are achieved.

Patients and methods: One hundred patients (7 males, 93 females; median age 57 years; range 31 to 76 years) with established RA receiving any conventional synthetic disease modifying anti-rheumatic drug (DMARD) and/or biological DMARD for at least three months were enrolled. Disease activity was determined by using the Simplified Disease Activity Index. First, patients were categorized into four groups as remission, low disease activity, moderate disease activity, and high disease activity. Then, they were divided into two subgroups, namely a remission/low disease activity subgroup and moderate disease activity/high disease activity subgroup.

Results: Fifty-one percent of the patients had remission or low disease activity. The most frequently used conventional synthetic DMARDs were methotrexate $(50 \%)$ and leflunomide (34\%). Forty-five percent of patients were receiving glucocorticoid therapy. In patients receiving only conventional synthetic DMARDs, the proportion of remission and low disease activity was 54\% (42/78). Forty-two percent (8/19) of the patients receiving biological DMARDs were in remission or had low disease activity. A comparison of subgroups revealed that median age and sulfasalazine use were significantly higher in the moderate disease activity/high disease activity subgroup.

Conclusion: The results of this study demonstrated that half of patients with established RA had moderate or high disease activity in our local outpatient clinic. Some barriers might be responsible for the difficulties in controlling disease activity. Determining such barriers might result in a better clinical response during the management of patients with established RA in real-life practice.

Keywords: Disease activity; disease modifying anti-rheumatic drug; established rheumatoid arthritis.
\end{abstract}

In light of advances in rheumatoid arthritis (RA) treatment, therapeutic decision making is now based on the concept of treat-to-target, and the target is to achieve remission. ${ }^{1}$ Although remission is the leading target, low disease activity (LDA) is also considered an acceptable disease activity status in patients with longstanding RA. ${ }^{2}$

The real-life data of patients with RA suggest that a considerable proportion of patients do not achieve disease remission or LDA..$^{2,3}$ This statement is also true for Turkish RA patients who participated in a multicenter study based on the RA registry of the Turkish League Against Rheumatism. ${ }^{4}$ The proportion of remission or LDA for each center was not reported in this multicenter study. The first step to avoid barriers to achieving treatment target in RA patients may be to reveal rate of treatment success in a rheumatic disease clinic. Thus, in this study, we aimed to explore the current disease activity status and simultaneous pharmacological therapies in patients with established RA to determine the extent to which treatment targets are achieved. 


\section{PATIENTS AND METHODS}

A total of 100 patients (7 males, 93 females; median age 57 years; range 31 to 76 years) with established RA were included in this observational, cross-sectional study. The study was approved by the local ethical committee (decision number: 8/10.02.2015).

Rheumatoid arthritis patients with a disease duration longer than 12 months, who had been taking a conventional synthetic disease-modifying antirheumatic drug (csDMARD) and/or biological disease-modifying antirheumatic drug (bDMARD) for at least three months, and had a recorded visit within the previous three months at the time of data collection were enrolled. Demographic characteristics of the patients, disease duration, tender and swollen joint count, patient and provider global assessment of disease activity, and $\mathrm{C}$-reactive protein level were recorded.

The Simplified Disease Activity Index (SDAI) was used to measure the most-recent disease activity. Patients were categorized into four groups according to the SDAI scores as follows: remission, LDA, moderate disease activity (MDA), and high disease activity (HDA). Threshold values for remission, LDA, and HDA were 3.3, 11, and 26 , respectively. ${ }^{5}$ Patients with missing data for any of the variables required to calculate the SDAI were excluded from the analysis. Thereafter, patients were categorized into two subgroups as remission/LDA subgroup and MDA/HDA subgroup.

\section{Statistical analysis}

MedCalc Statistical Software version 14.12.0 (MedCalc Software buba, Ostend, Belgium) was used for statistical analysis. The two subgroups were compared in terms of disease duration, age, medications, and each dimension of the SDAI. In the comparison of the subgroups, Mann-Whitney $\mathrm{U}$ test was used for numerical variables, and the continuity correction chi-square test or Fisher's exact test was used for categorical variables. To calculate the minimum required sample size ( $\alpha$ : 0.05 , power: $80 \%$ ), we used the results of the study of Bal et al. ${ }^{4}$ To the best of our knowledge, the study of Bal et al. ${ }^{4}$ which was based on the Turkish League Against Rheumatism RA registry data had the largest sample size of those conducted in Turkey, with 2,359 participants and mean disease duration of $11.9 \pm 8.7$ years. In the same study, ${ }^{4}$ the overall proportion of patients with remission and LDA was 32\% $(n=744)$. When the expected rate of remission/LDA was determined as $50 \%$, the minimum required sample size was 61 . In the cases of $35 \%, 40 \%$, and $45 \%$ of the expected rate, the minimum required sample sizes were $1,980,294$, and 115 , respectively. There were some limitations when it came to calculating sample size. While we evaluated disease activity via the SDAI, Bal et al. ${ }^{4}$ used the Disease Activity Score with 28 joints including the erythrocyte sedimentation rate. Using diverse indices to evaluate the disease activity may lead to incorrect sample size estimation because of discrepancies between the indices.

\section{RESULTS}

The median disease duration was 120 (range 12 to 420 ) months. In the comparison of subgroups, while the median age was significantly higher in the MDA/HDA subgroup $(p=0.031)$, disease durations were comparable $(p=0.992)$. The median SDAI score was 11 (range 0 to 57.5). Demographic and clinical characteristics of patients are presented in Table 1.

The overall percentage of patients classified in the remission/LDA group was 51\% (Figure 1). Seventy-eight percent of all patients were receiving monotherapy or combination therapy with csDMARD, 19\% were receiving bDMARD (as monotherapy or combined with csDMARD), and 3\% were receiving only glucocorticoid therapy.

The percentages of patients receiving methotrexate (MTX) and leflunomide (LEF) monotherapy within the csDMARD group were $39 \%$ and $28 \%$, respectively. Twenty-five percent of 78 patients were being treated with combination therapy. The most-used combination therapies were MTX and sulfasalazine combination and MTX and LEF combination (Figure 2). Fifty-four percent of these 78 patients receiving csDMARD were in remission or had LDA status.

Of a total of 100 patients, 50\% were being treated with MTX with a median dose of $15 \mathrm{mg}$ /week (range 7.5 to 20), and $45 \%$ were being treated with methyl prednisolone with a 
Table 1. Demographic and clinical characteristics of total patient population and each subgroup

\begin{tabular}{|c|c|c|c|c|c|c|c|c|c|c|c|c|c|}
\hline & \multicolumn{4}{|c|}{ Total study population $(\mathrm{n}=100)$} & \multicolumn{4}{|c|}{ Remission/LDA $(n=51)$} & \multicolumn{4}{|c|}{ MDA/HDA $(n=49)$} & \multirow[b]{2}{*}{$p$} \\
\hline & $\mathrm{n}$ & $\%$ & Median & Range & $\mathrm{n}$ & $\%$ & Median & Range & $\mathrm{n}$ & $\%$ & Median & Range & \\
\hline Age (years) & & & 57 & $31-76$ & & & 54 & $31-76$ & & & 60 & $37-76$ & 0.031 \\
\hline \multicolumn{14}{|l|}{ Gender } \\
\hline Female & 93 & 93 & & & 48 & 94 & & & 45 & 92 & & & 0.712 \\
\hline Disease duration (months) & & & 120 & $12-420$ & & & 120 & $12-408$ & & & 120 & $12-420$ & 0.992 \\
\hline Tender joint count & & & 2 & $0-28$ & & & 0 & $0-9$ & & & 7 & $0-28$ & $<0.0001$ \\
\hline Swollen joint count & & & 0 & $0-24$ & & & 0 & $0-2$ & & & 1 & $0-24$ & $<0.0001$ \\
\hline PGDAE, VAS $(0-10 \mathrm{~cm})$ & & & 4 & $0-10$ & & & 1 & $0-5$ & & & 5.5 & $2-10$ & $<0.0001$ \\
\hline PrGDAE, VAS $(0-10 \mathrm{~cm})$ & & & 3 & $0-9$ & & & 1 & $0-5$ & & & 5 & $1-9$ & $<0.0001$ \\
\hline $\mathrm{CRP}(\mathrm{mg} / \mathrm{dL})$ & & & 0.52 & $0.02-6.18$ & & & 0.46 & $0.02-6.18$ & & & 0.69 & $0.05-5.5$ & 0.023 \\
\hline SDAI & & & 11.0 & $0-57.5$ & & & 5.7 & $0-11$ & & & 21.2 & $11.2-57.5$ & $<0.0001$ \\
\hline
\end{tabular}

LDA: Low disease activity; MDA: Moderate disease activity; HDA: High disease activity; PGDAE: Patient global disease activity evaluation; VAS: Visual analogue scale; PrGDAE: Provider global disease activity evaluation; CRP: C-reactive protein; SDAI: Simplified disease activity index.

median dose of $4 \mathrm{mg} /$ day (range 1 to 16). The number of patients receiving sulfasalazine was significantly higher in patients with MDA/HDA $(p=0.046)$. The frequencies of patients receiving DMARD according to disease activity state are presented in Table 2.

The most prescribed bDMARDs were tumor necrosis factor alpha inhibitors ( $\mathrm{n}=15,79 \%)$. The other prescribed bDMARDs were abatacept in one patient and rituximab in three patients. Of 19 patients treated with bDMARDs, 42\% $(\mathrm{n}=8)$ were receiving additional csDMARDs. MTX was the most prescribed csDMARD by $75 \%$ $(n=6)$. Forty-two percent of the patients receiving bDMARDs were in the remission/LDA subgroup. The median age of the bDMARD receiving population was 55 years (range 34 to 68) in the remission/LDA subgroup and 59 years (range 43 to 65 ) in the MDA/HDA subgroup $(p=0.934)$. While three out of eight patients receiving bDMARD were using glucocorticoid

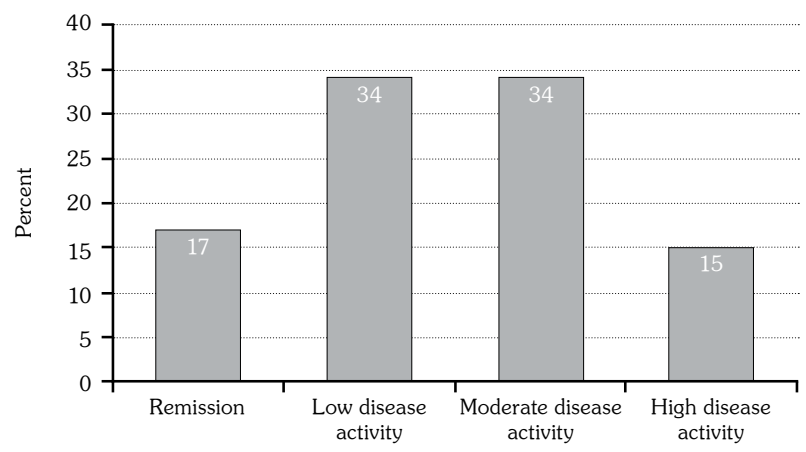

Figure 1. Proportions of patients in each disease activity category. concomitantly in the remission/LDA subgroup, five out of 11 patients receiving bDMARD were using glucocorticoid concomitantly in the MDA/ HDA subgroup, but there was no significant difference $(p=1.000)$.

\section{DISCUSSION}

Since the characteristics of patients in reallife practice are generally different from those in clinical trials, making treatment decisions in routine clinical care is challenging., ${ }^{2,3}$ Our results demonstrated that a state of remission or LDA was achieved in half of the patients in our real-life clinical practice. MTX and LEF were the most frequently preferred csDMARDs. Nineteen percent of patients were receiving bDMARDs; of these patients, only $42 \%$ were in the remission/LDA subgroup.

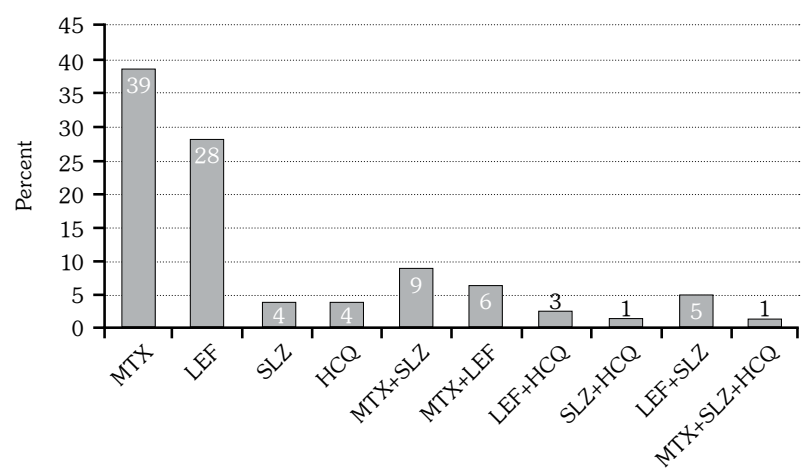

Figure 2. Pharmacological therapy percentages of 78 patients receiving conventional synthetic diseasemodifying anti-rheumatic drug. MTX: Methotrexate; LEF: Leflunomide; SLZ: Sulfasalazine; HCQ: Hydroxychloroquine. 


\begin{tabular}{|c|c|c|c|c|c|c|c|c|c|c|c|c|c|}
\hline & \multicolumn{4}{|c|}{ Total study population $(\mathrm{n}=100)$} & \multicolumn{4}{|c|}{ Remission/LDA ( $\mathrm{n}=51)$} & \multicolumn{4}{|c|}{ MDA/HDA $(n=49)$} & \multirow[b]{2}{*}{$p$} \\
\hline & $\mathrm{n}$ & $\%$ & Median & Range & $\mathrm{n}$ & $\%$ & Median & Range & $\mathrm{n}$ & $\%$ & Median & Range & \\
\hline Methyl prednisolone & 45 & 45 & & & 18 & 35 & & & 27 & 55 & & & 0.074 \\
\hline $\begin{array}{l}\text { Glucocorticoid dosage } \\
\text { (mg/day) }\end{array}$ & & & 4 & $1-16$ & & & 4 & $1-8$ & & & 4 & $2-16$ & 0.169 \\
\hline Methotrexate use & 50 & 50 & & & 28 & 55 & & & 22 & 45 & & & 0.424 \\
\hline $\begin{array}{l}\text { Methotrexate dosage } \\
\text { (mg/week) }\end{array}$ & & & 15 & $7.5-20$ & & & 10 & $7.5-20$ & & & 15 & $10-20$ & 0.096 \\
\hline Leflunomide & 34 & 34 & & & 17 & 33 & & & 17 & 35 & & & 1.000 \\
\hline Sulfasalazine & 16 & 16 & & & 4 & 8 & & & 12 & 25 & & & 0.046 \\
\hline Hydroxychloroquine & 7 & 7 & & & 3 & 6 & & & 4 & 8 & & & 0.712 \\
\hline bDMARD & 19 & 19 & & & 8 & 16 & & & 11 & 22 & & & 0.544 \\
\hline csDMARD combination & 20 & 20 & & & 6 & 12 & & & 14 & 29 & & & 0.064 \\
\hline
\end{tabular}

Although the primary target is remission in the management of early RA, the achievement of remission or LDA is also acceptable in longstanding, established RA. In particular, achieving a better clinical outcome in terms of favorable functional improvement and preventing the progression of the structural damage of affected joints are important in clinical practice. ${ }^{6,7} \mathrm{LDA}$ is associated with favorable functional outcomes and less structural damage than MDA and HDA, but with worse outcomes than remission. ${ }^{2}$

Our study sample consisted of patients with established RA. We determined that overall remission and LDA were achieved in half of the study sample. In two reports from a developed country, ${ }^{8,9}$ the proportions of patients with MDA and HDA were lower than ours, but still quite high by $39 \%$ (number of study population $=5,686$ ) and $37.5 \%$ (number of study population $=4,037$ ), respectively. In a report on a multicenter study from Turkey that used the Turkish League Against Rheumatism RA registry data, ${ }^{4}$ the overall proportion of patients with MDA and HDA was reported to be $68 \%$ (number of study population=2,539).

Some barriers to the control of disease activity have been identified. Comorbidities, older age, irreversible joint damage, patientdriven preference, safety concerns, and resistant disease might hamper management of the disease..$^{9,10}$ Although not the focus of our study, some barriers including increased disease duration and inadequate medication were also identified. DMARDs may lose efficacy over time or patients may develop intolerance to them. ${ }^{10}$ Increased disease duration has been suggested to be associated with a poor clinical response to DMARDs, including MTX.11 In the present study, we also found that the proportion of patients receiving MTX decreased as the disease duration increased. This result might be associated with a lack of responsiveness to treatment by MTX and/or to adverse events related to MTX medication.

The performance of disease activity measurement tools used in RA in defining remission, in particular, is rather different. ${ }^{12}$ Although Disease Activity Score 28 is used for the prescription of bDMARDs in Turkey, in this study, disease activity was assessed using the SDAI, which is the most stringent index for defining remission. Both the American College of Rheumatology and European League Against Rheumatism recommend using the SDAI or a Boolean-based definition of remission. ${ }^{13}$ The dimension of 'patient global assessment of disease activity' of the composite indices used in RA may be influenced by non-inflammatory pain syndromes such as fibromyalgia and depression, particularly in patients with long-standing disease. ${ }^{9,10}$

The management of RA depends primarily on the use of DMARDs, ${ }^{2}$ MTX being the anchor drug. ${ }^{14}$ Maximization of treatment effects includes reaching an optimal MTX dose within a few weeks and maintaining the maximal dose (25-30 mg weekly) for at least eight 
weeks. ${ }^{15}$ In cases of MTX contraindications or early intolerance, LEF or sulfasalazine should be considered as part of the first treatment strategy. Combination therapy with csDMARDs should also include MTX. ${ }^{2}$ In our study, the main csDMARD used was MTX in accordance with the recommendations, the maximal dosage of MTX was not reached or maintained. Only five out of 50 patients were treated with the maximum MTX dosage of $20 \mathrm{mg} /$ week. Inadequate MTX dosage might be one of the barriers to optimal control of the disease activity for RA patients in our study. The second most frequently used csDMARD in our study was LEF.

In contrast to the European League Against Rheumatism recommendation, ${ }^{2}$ in which lowerdose glucocorticoids are considered as part of the initial treatment strategy for early RA, a considerable proportion of our patients, who all had established RA, were treated with glucocorticoids. The prescription of glucocorticoid increased as disease activity increased.

Although the ratio of remission or physical independence has increased with bDMARDs, treatment responses in most patients using these drugs do not meet the goal of good response or remission. ${ }^{16}$ Older age, low functional status, and concomitant prednisolone treatment have been suggested as negative predictors of a clinical response and remission. ${ }^{17}$ Our results demonstrated that $58 \%(\mathrm{n}=11)$ of the patients receiving bDMARDs had MDA or HDA status. These patients in the MDA/HDA subgroup did not exhibit differences from those receiving bDMARDs in remission/LDA subgroup in terms of age and concomitant glucocorticoid use.

This study has some limitations. Although our sample size was adequate to use the results of Bal et al. ${ }^{4}$ to calculate the appropriate number of patients, the minimum required size might change if the data of another RA registry were used. In a study population consisting of patients with early $\mathrm{RA}$, the proportion of medications used and the proportion of the patients in each disease activity category may differ. Although one of the inclusion criteria was that the same medication must have been used for at least three months, we did not include data on how much time had elapsed after the onset of treatment. For a patient with HDA, receiving the same medication for three months may not be adequate when it comes to evaluating the usefulness of the treatment if there is a moderate response to it. Disability may lead to higher disease activity measurement despite the low inflammation. Lack of information about the functional status of the patients is another limitation of our study.

In conclusion, the results of this study demonstrated that a significant part of the patients with established RA was not in the state of remission or LDA. The MTX dosage was lower than that in the recommendations. Moreover, the treatment duration for glucocorticoid was quite long. More than half of the patients receiving bDMARDs still had MDA or HDA. In addition, some patients were treated with csDMARDs and glucocorticoids despite needing biological treatment. Even if a therapeutic decision is based on the concept of treat-to-target in the recommendations, the concept of treat-to-symptom is still prevalent in real-life practice. When considered all together, there is an unmet need for treatment, which may result from some barriers to effective therapy. Determining the proportion of patients achieving the treatment target in established RA might be the first step in identifying and avoiding these barriers. Further studies with the aim of determining the population specific barriers that are responsible for higher disease activity might provide the clinicians with a strategy to take precautions during RA management.

\section{Declaration of conflicting interests}

The authors declared no conflicts of interest with respect to the authorship and/or publication of this article.

\section{Funding}

The authors received no financial support for the research and/or authorship of this article.

\section{REFERENCES}

1. Smolen JS, Landewé R, Breedveld FC, Dougados M, Emery P, Gaujoux-Viala C, et al. EULAR recommendations for the management of rheumatoid arthritis with synthetic and biological diseasemodifying antirheumatic drugs. Ann Rheum Dis 2010;69:964-75.

2. Smolen JS, Landewé R, Breedveld FC, Buch $M$, Burmester G, Dougados $M$, et al. EULAR recommendations for the management of rheumatoid 
arthritis with synthetic and biological diseasemodifying antirheumatic drugs: 2013 update. Ann Rheum Dis 2014;73:492-509.

3. Mierau M, Schoels M, Gonda G, Fuchs J, Aletaha D, Smolen JS. Assessing remission in clinical practice. Rheumatology (Oxford) 2007;46:975-9.

4. Bal A, Ataman Ş, Bodur H, Rezvani A, Paker N, Taştekin N, et al. Characteristics of patients with rheumatoid arthritis in Turkey: results from the Turkish Against Rheumatism Rheumatoid Arthritis Registry. Arch Rheumatol 2015;30:16-22.

5. Smolen JS, Breedveld FC, Schiff MH, Kalden JR, Emery P, Eberl G, et al. A simplified disease activity index for rheumatoid arthritis for use in clinical practice. Rheumatology (Oxford) 2003;42:244-57.

6. Aletaha D, Smolen JS. Joint damage in rheumatoid arthritis progresses in remission according to the Disease Activity Score in 28 joints and is driven by residual swollen joints. Arthritis Rheum 2011;63:3702-11.

7. Gärtner M, Mandl P, Radner H, Supp G, Machold KP, Aletaha D, et al. Sonographic joint assessment in rheumatoid arthritis: associations with clinical joint assessment during a state of remission. Arthritis Rheum 2013;65:2005-14.

8. Littlejohn G, Roberts L, Arnold M, Bird P, Burnet S, de Jager $\mathrm{J}$, et al. A multi-center, observational study shows high proportion of Australian rheumatoid arthritis patients have inadequate disease control. Int J Rheum Dis 2013;16:532-8.

9. Tymms K, Zochling J, Scott J, Bird P, Burnet S, de Jager $\mathrm{J}$, et al. Barriers to optimal disease control for rheumatoid arthritis patients with moderate and high disease activity. Arthritis Care Res (Hoboken) 2014;66:190-6.

10. Horton SC, Walsh CA, Emery P. Established rheumatoid arthritis: rationale for best practice: physicians' perspective of how to realise tight control in clinical practice. Best Pract Res Clin Rheumatol
2011;25:509-21.

11. Anderson JJ, Wells G, Verhoeven AC, Felson DT. Factors predicting response to treatment in rheumatoid arthritis: the importance of disease duration. Arthritis Rheum 2000;43:22-9.

12. Fleischmann $\mathrm{R}$, van der Heijde D, Koenig AS, Pedersen R, Szumski A, Marshall L, et al. How much does Disease Activity Score in 28 joints ESR and CRP calculations underestimate disease activity compared with the Simplified Disease Activity Index? Ann Rheum Dis 2015;74:1132-7.

13. Methotrexate for the treatment of rheumatoid arthritis in the biologic era: still an "anchor" drug? American College of Rheumatology/European League Against Rheumatism provisional definition of remission in rheumatoid arthritis for clinical trials. Arthritis Rheum 2011;63:573-86.

14. Favalli EG, Biggioggero M, Meroni PL. Methotrexate for the treatment of rheumatoid arthritis in the biologic era: still an "anchor" drug? Autoimmun Rev 2014;13:1102-8.

15. Visser K, van der Heijde D. Optimal dosage and route of administration of methotrexate in rheumatoid arthritis: a systematic review of the literature. Ann Rheum Dis 2009;68:1094-9.

16. Listing J, Strangfeld A, Rau R, Kekow J, GromnicaIhle $\mathrm{E}$, Klopsch $\mathrm{T}$, et al. Clinical and functional remission: even though biologics are superior to conventional DMARDs overall success rates remain low--results from RABBIT, the German biologics register. Arthritis Res Ther 2006;8:66.

17. Hetland ML, Christensen IJ, Tarp U, Dreyer L, Hansen A, Hansen IT, et al. Direct comparison of treatment responses, remission rates, and drug adherence in patients with rheumatoid arthritis treated with adalimumab, etanercept, or infliximab: results from eight years of surveillance of clinical practice in the nationwide Danish DANBIO registry. Arthritis Rheum 2010;62:22-32. 